\title{
Performance, performatividad y memoria
}

\author{
Performance, performativity, and memory
}

Diana Paola Triana Moreno ${ }^{1}$

Pontificia Universidad Javeriana, Colombia

Recepción: 23 de febrero del 2017

Evaluación: 14 de mayo del 2018

Aceptación: 3 de junio del 2018

1 Candidata a doctora en Filosofía por la Pontificia Universidad Javeriana y miembro del grupo de investigación Problemas de filosofía de la misma universidad. Magíster en Estudios Sociales de la Universidad Pedagógica Nacional. Filósofa de la Universidad Libre e historiadora de la Universidad del Rosario. Sus intereses académicos están vinculados con la filosofía estética, la fenomenología, la historia del arte y los estudios de género y feminismos.

Correo electrónico: trianam.diana@gmail.com; diana.triana@javeriana.edu.co 


\title{
Resumen
}

El objetivo del presente artículo de reflexión es hacer evidente la relación entre la categoría de performatividad en Judith Butler y el arte de la performance como una aproximación a la construcción de la memoria. Por un lado, el arte de la performance ofrece un problema en torno al registro, al archivo y a la memoria de la acción artística al considerar que el acto es irrepetible, único y fugaz. Esta discusión está vinculada con la reperformación como alternativa de memoria que conserva la naturaleza de esta práctica artística. Por otro lado, de acuerdo con Butler, la identidad es un efecto de una serie de performances regulados, esto revela cómo la performatividad sugiere un tipo de identidad no esencial donde la memoria está siempre abierta a nuevas inscripciones cuyo soporte está en la reiteración de actos discontinuos. En consecuencia, la repetición de los actos que propone Butler permite comprender que en la reperformance existe otra forma para abordar una memoria restaurada.

Palabras clave: performatividad, performance, memoria, identidad, repetición.

\begin{abstract}
The aim of this article of reflection is to show the relationship between the category of performativity in Judith Butler and the art of performance as an approach to memory construction. On one hand, the art of performance offers a problem concerning the register, the file, and the memory of the artistic action by considering that the act is unrepeatable, unique, and transient. This discussion is linked with the re-performation as an alternative to the memory that keeps the nature of this artistic practice. On the other hand, according to Butler, the identity is an effect of a sequence of regulated performances, which reveals how the performativity suggest a kind of identity, which is not essential when the memory is always open to new inscriptions and whose support is in the reiteration of discontinuous acts. Consequently, the repetition of the acts that Butler proposes allows to understand that, in the re-performance, there exists another way to see a restored memory.
\end{abstract}

Keywords: performativity, performance, memory, identity, repetition. 


\section{Performance, performativité et mémoire}

\section{Résumé}

L'objectif du présent article de réflexion, est de mettre en évidence la relation entre la catégorie de performativité chez Judith Butler et l'art de la performance en tant que rapprochement à la construction de la mémoire. D'une part, l'art de la performance évoque un problème autour du registre, de l'archivage et de la mémoire de l'action artistique, en considérant que l'acte est non reproductible, unique et éphémère. Cette discussion est liée à la reperformation comme alternative de mémoire qui préserve la nature de cette pratique artistique. D'autre part, d'après Butler, l'identité est un effet d'une série de performances régulées, ce qui révèle de quelle façon la performativité suggère un type d'identité non essentielle où la mémoire est toujours ouverte à des nouvelles inscriptions dont le support est la réitération d'actes discontinus. Par conséquent, la répétition des actes que propose Butler permet de comprendre que dans la reperformance il existe une façon autre d'aborder une mémoire restaurée.

Mots-clés: performativité, performance, mémoire, identité, répétition.

\section{Performance, performatividade e memória}

\section{Resumo}

O objetivo do presente artigo de reflexão é evidenciar a relação entre a categoria de performatividade em Judith Butler e a arte da performance como uma aproximação à construção da memória. Por um lado, a arte da performance oferece um problema em torno do registro, do arquivo e da memória do agir artístico ao considerar que o ator é irrepetível, único e fugaz. Essa discussão está vinculada à reperformação como alternativa de memória que conserva a natureza dessa prática artística. Por outro lado, segundo Butler, a identidade é um efeito de uma série de performances reguladas. Isso revela como a performatividade sugere um tipo de identidade não essencial, na qual a memória está sempre aberta a novas inscrições cujo suporte está na reiteração dos atos descontínuos. Consequentemente, a repetição dos atos que propõe Butler permite compreender que na reperformance existe outra forma para abordar uma memória restaurada.

Palavras-chave: performatividade, desempenho, memória, identidade, repetição. 


\section{Introducción}

Desde que Peggy Phelan (2011) introdujo la idea de la "Ontología del performance", para referirse al arte de la "representación sin reproducción", las discusiones en torno a la desaparición en el acto y la imposibilidad de repetición de la ejecución se han convertido en tópicos de una inagotable reflexión filosófica. Si la performance como acto solo se completa con su desaparición queda claro que cualquier iniciativa por repetir, registrar o reproducir la práctica será contraria a su naturaleza. Las consecuencias de esta postura han implicado múltiples discusiones en los Performance Studies en torno a las relaciones entre originales, copias, soportes, registros, archivos y repertorios, tratando de pensar la experiencia corporal y vivencial de la performance (Fuentes \& Taylor, 2011; Jones, 2011; Phelan, 2011; Schechner, 2011; Scheneider, 2011; Taylor, 2015). Sin lugar a duda este debate está lejos de concluir o llegar a un acuerdo mayoritario sobre estos temas de indagación. Las posiciones son variadas, desde aquellos que, como Phelan, consideran que es contrario a la naturaleza de la performance buscar la manera de capturar el acto sobre algún tipo de soporte documental, tanto como en considerar que la repetición de la performance anula su potencia como arte de acción presente. Por el contrario, otros autores consideran que la documentación y archivo sobre la práctica artística es vital, no solo para efectos de construir la historia de la práctica artística, sino para no perder en lo efímero los esfuerzos y las apuestas políticas que se juegan desde los cuerpos. La necesidad del registro y la fidelidad de éste a la práctica de la performance son cuestiones transversales para los análisis estéticos contemporáneos que deben ser examinadas a profundidad.

Este artículo de reflexión pretende establecer un puente entre la propuesta performativa de Judith Butler y la ontología de la performance. En este sentido, se busca discutir las posibilidades de la reperformación y los horizontes con que este tipo de repertorio contribuye en la construcción de memoria incorporada. En el fondo, se busca presentar una solución alternativa a la forma de entender la memoria, la repetición diferencial y la restauración. Así, vale la pena preguntar ¿cómo se construye la memoria cuando se considera que cada acontecimiento es único e irrepetible y no admite registro?, ¿cómo entender la mediación que supone la memoria sobre un acontecimiento pasado?, ¿qué alternativas existen para ser coherentes con la naturaleza del arte de la performance y no renunciar a documentar la ejecución? 
El artículo está estructurado en cuatro partes: en la primera sección, se muestra la forma en la que la performance ha sido comprendida como un arte efímero, presente, irrepetible y sin registro. En las siguientes dos secciones, se indagará desde Butler y Derrida las posibilidades de comprender la memoria subjetiva y las formas en que la reiteración estilizada de actos y la repetición desigual muestran la tensión permanente entre lo mismo y lo otro. Se evidencia que la memoria siempre está elaborándose y en cada reiteración existe un desplazamiento. Luego, en la última sección, se explica la importancia de comprender la performance y la reperformance como una memoria restaurada, propuesta que está en la misma línea analítica de Butler y Derrida. En consecuencia, se argumenta que la reperformación es la única manera de mantener la performance en un horizonte de tradición, esto es, la memoria restaurada de la performance se debe actuar y reactivar para mantenerse viva.

\section{La memoria se desvanece en el acto}

Durante los últimos años el arte de la performance ha cobrado un lugar central dentro del ámbito artístico. La apuesta por hacer del acto la obra de arte ha supuesto un desafío, en la medida en que no hay posibilidad de soportar, registrar y reproducir la performance sin perder al mismo tiempo su esencia. La documentación del acto es un problema nuclear en la teoría del arte en relación con la posibilidad de establecer una memoria/archivo sobre éste, pues el acto es fundamentalmente un arte del presente; la performance es irrepetible y es fascinante porque es única y efímera. Eso supone que, una vez terminado el acto, el único medio para acceder a éste es a través de las huellas que ha dejado (Scheneider, 2011).

Si bien los registros fotográficos, multimediales, entrevistas a los miembros de la audiencia, los curadores y los mismos artistas de la exhibición hacen parte del corpus documental de la puesta en escena, estos presentan limitaciones sobre el acontecimiento artístico que son incomparables, en cierto sentido, con la experiencia vivida, que es igualmente limitada (Banks, 2014; Reason, 2003). Cada uno de esos registros es simplemente un escorzo, es decir, una perspectiva parcial de la ejecución artística que cambia la naturaleza misma de este arte, en tanto que en la performance desaparece en medio de su acto. Por ello, siempre que el acto quede en el registro fotográfico o multimedia, éste habrá desaparecido para siempre, pues la performance es ante todo el arte del presente: "La única vida del performance transcurre en el presente, el performance no se guarda, registra, documenta ni participa de 
manera alguna en circulación de las representaciones: una vez que lo hace, se convierte en otra cosa; ya no es performance" (Phelan, 2011, p. 97).

El desafío es aún mayor cuando se busca repetir una performance cuya repetición es imposible. La reperformance debe valerse necesariamente de las huellas dejadas en los registros ante la imposibilidad de tener acceso directo a la performance misma. En este sentido, la performance es incomparable a su documentación, puesto que ésta nunca hará justicia a la ejecución y a la experiencia de aquella. De acuerdo con Marina Abramovic, la única manera de documentar las piezas artísticas es volviendo a realizarlas:

A menudo tenía que confiar en el testimonio de los espectadores, videograbaciones y fotografías de mala calidad. Debido a las condiciones extremas de la documentación artística de las performances, estos soportes sustitutos nunca hicieron justicia a la ejecución real. La única forma real de documentar las piezas de arte es volver a interpretar las piezas² (Abramovic, 2005, p. 11. La traducción es mía).

La documentación de una pieza de performance es la reperformance de la pieza misma, cuestión que es ciertamente cuestionable, en tanto la repetición del acto no es sino una citación o reiteración de una acción que ya no puede contar con las condiciones para asegurar que sea la misma performance.

El problema de la memoria se revela con claridad en la performance Seven Easy Pieces presentada por Abramovic en el Museo Guggenheim de Nueva York en el 2005. Se trata de un circuito de reperformance en las que la artista se esfuerza por recuperar algunas de las piezas más emblemáticas de los años 1960 y $1970^{3}$. Estos actos dejaron un registro fragmentado por su condición de arte efímero, dado que el arte de la performance, durante su época temprana, renunciaba y rechazaba la posibilidad de registrar sus ejecuciones fundamentado en un interés político por cuestionar el espacio institucionalizado del museo y la comercialización del arte (Jones, 2011; Tomic, 2008).

Texto original en inglés "one often had to rely on testimonial witnesses, poor quality video recordings, and photos negatives. Due to the dire conditions of performances art documentation, these substitutable media never did justice to the actual performance. The only real way to document a performances art pieces is to reperform the pieces itself".

3 Bruce Nauman, Body Pressure (1974); Vito Acconci, Seedbed (1972); Valie Export, Action Pants: Genital Panic (1969); Gina Pane, The Conditioning (1973); Joseph Beuys, How to Explain Pictures to a Dead Hare (1965); Abramovic, Lips of Thomas (1975) y Entering the Other Side (2005) (Abramovic et al., 1998). 
Si el arte se desplazó y privilegió al cuerpo como lugar de producción fue por el interés en impedir la existencia de un arte objetual proclive a las demandas del mercado. De acuerdo con Amelia Jones (2011), concebir el cuerpo como un medio de expresión en el arte de la performance, implica una exploración y problematización tanto de la comercialización de éste dentro del sistema capitalista como del ocultamiento que del cuerpo se había hecho durante la Modernidad. En este sentido, las producciones de arte corporal son producto de la intersubjetividad, pues éstas no se agotan en lo que se exhibe, sino que se completa en el deseo del espectador: "el cuerpo no es aquí un mero objeto expuesto a la mirada consumista del espectador, sino el lugar de producción y negociación de lo social" (Fuentes, 2011, p. 126).

Por tanto, las representaciones no son el resultado de una subjetividad creativa y corpórea que está al margen de los otros. Lo que Abramovic sugiere con Seven Easy Pieces es revivir las performances, reestablecer los lazos con la tradición inicial y configurar unas huellas materiales sobre la ejecución. Aunque la artista reconoce que la nueva puesta en escena implica una experiencia distinta en tanto que toda repetición supone una reelaboración, también considera que solo reperformando es posible dar un lugar a la performance dentro de la historia del arte al indagar por su tradición. Sin embargo, puede ocurrir que en medio de la reperformación se cambie la naturaleza de la propuesta inicial de la performance y dé lugar a otros resultados.

En el caso de la obra Body Pressure, el cambio de naturaleza de la propuesta es evidente. La iniciativa de Bruce Nauman (1974) consistió en elaborar un texto con instrucciones precisas que el espectador debía ejecutar usando su propio cuerpo y la superficie arquitectónica. Allí se jugaba la experiencia física, las limitaciones del cuerpo y de la mente, tal como se muestra en este fragmento:

(...) Consider the parts of your back which/ press against the wall; press hard and/ feel how the front and back of your/ body press together/ Concentrate on the tension in the muscles/pain where bones meet, fleshy deformations that occur under pressure; consider/body hair, perspiration, odors (smells)/This may be a very erotic exercise (Nauman, 1974, p. 125).

En esta performance solo se disponía de una torre de papeles rosados con las instrucciones impresas en ellos. En contraste con la reperformance de Abramovic en el año 2005, en la cual la artista ya no dejó en los espectadores la labor de revivir la propuesta de Nauman, dado que ella misma se encargó de ejecutarla. Si bien la reperformance de Abramovic permitió hacer presente la 
performance de Nauman, hubo en su interpretación un cambio sustancial de la naturaleza, no solo de la relación del artista con los espectadores, sino también de la naturaleza efímera y presente, corporal y experiencial de la obra.

Sin embargo, la documentación de la performance a través de la reperformación es solo un estímulo para la memoria, un dispositivo para que el recuerdo se haga presente. Si la performance es el resultado de la experiencia compartida entre el artista y los espectadores, es necesario asumir que si estos cambian la performance también lo hará (Beisenbach, 2010). Sin embargo, la muestra de Abramovic, aunque se elabora directamente desde la documentación disponible de los originales, convierte esa reperformance en una performance original. Una vez instalada en la escena 'Seven easy pieces' se elabora con ello el olvido de las imágenes originales, en la que Abramovic ha efectuado un desplazamiento y reinaugurado la performance (Banks, 2014)

La idea de la ontología de la performance, definida como el arte de la 'representación sin reproducción', evidencia que la performance es un acto que solo es posible realizar una única vez, teniendo en cuenta que este arte solo llega a ser lo que es a través de su desaparición. En este sentido, Peggy Phelan (2011) cuestiona las formas de documentación y archivo del arte corporal, al ser simplemente formas reproductivas que son radicalmente distintas a la performance, es decir, que toda documentación es solo un registro de la performance y no la performance. De acuerdo con Phelan, hacer performance es situarse en el ahora, otorgar valor al presente, incluso cuando éste se repite; la repetición no es más que una nueva performance que debe ser considerada en su singularidad con independencia absoluta del referente anterior, no puede ser nunca una reproducción, a diferencia de las insinuaciones de Abramovic relativas a la reperformación como una alternativa ante lo efímero de esta forma de arte.

En consecuencia, una performance siempre será un original, incluso, la repetición se manifestará como un trabajo original cada vez distinto al acto performático de origen, de modo que cada repetición será un original. Aquí se revela la paradoja en la que la repetición no se muestra bajo el signo de lo mismo, sino de lo Otro. La relación entre original y copia en la performance siempre será objeto de indagación, pues unos serán independientes de otros; el acto se altera en su reiteración, borrando su originalidad e instaurándola simultáneamente: lo mismo se presentará siempre distinto de sí. En el fondo de esta discusión emerge la repetición que oculta un desplazamiento diferencial, 
de modo que la repetición se sitúa en la performance como la identidad de lo no idéntico: "hago, rehago y deshago mis concepciones a partir de un horizonte móvil, de un centro siempre descentrado, de una periferia siempre desplazada que los repite y los diferencia" (Deleuze, 2002, p. 17).

En ese sentido, el arte de la performance y la noción de performatividad pueden ser equiparadas en referencia a la producción de memoria. La preocupación por el soporte documental de la performance encuentra en la idea de performatividad de Judith Butler una categoría analítica interesante para comprender la repetición y la reiteración de actos discontinuos en la producción de la memoria. Si bien Butler indaga por la construcción de la identidad de género, su reflexión es pertinente para considerar la conexión entre la discontinuidad del acto y la memoria del sujeto. Si asumimos que cada reelaboración y reactuación de la identidad puede llegar a ser una nueva inscripción que transforma la narrativa que el sujeto hace de sí mismo, entonces la producción de registros o huellas de memoria sobre lo vivido siempre deberá afrontarse a las nuevas versiones, en la que el sujeto es el productor de una narración siempre sobrepuesta a la anterior, que la borra mientras se resitúa.

\section{La memoria: un juego de varias piezas}

Un elemento central de la teoría de la performatividad propuesta por Judith Butler está íntimamente ligado a la perspectiva foucaultiana en torno a la naturaleza sociohistórica de la agencia, lo que significa comprender el 'Yo' construido por la historia y no simplemente situado en ella (Barthold, 2014). De acuerdo con esta visión, los sujetos instituyen acciones que son, al mismo tiempo, producto de instituciones previas, es decir, que las acciones siempre están determinadas por un horizonte de posibilidades más o menos restringidas y en las que el sujeto asume su propia capacidad de actuar dentro un teatro de operaciones (Butler \& Scott, 1992).

La identidad, entendida en esta perspectiva, se muestra como una posicionalidad que tiene su base en los múltiples discursos que circulan dentro de lo social, definiéndola y constituyéndola. Se trata siempre de un juego en la tensión entre lo instituyente y lo instituido, entre lo dado y lo imaginado. En este sentido, se trata de una identidad abierta y flexible que hace uso de estrategias e instrumentos provenientes de la tradición, a través de las estructuras culturales e históricas. La performatividad es la creación y el mantenimiento de una identidad mediante la repetición o reiteración de determinadas narrativas 
y prácticas que el sujeto agencia, es la puesta en escena de un rito que se dinamiza mediante comportamientos, conductas y actos a lo largo de la experiencia vital. Por ello, el acto performativo enuncia un fenómeno, mientras en la misma operación lo produce al nombrarlo y renombrarlo, actuarlo y reactuarlo (Brant, 2013). Así lo presenta Butler en su ensayo "Fundamentos contingentes: el feminismo y la cuestión del "postmodernismo":

"yo" — y no evado el pronombre - represento y resignifico las posiciones teóricas que me han constituido, trabajando con las posibilidades de su convergencia, y tratando de tomar en cuenta las posibilidades que sistemáticamente excluyen. Pero está claro que no es el caso que "yo" presido sobre las posiciones que me han constituido, reacomodándolas instrumentalmente, haciendo algunas a un lado, incorporando otras, aunque parte de mi actividad pueda tomar esa forma. El "yo" que seleccionaría entre ellas es siempre previamente constituido por ellas. El "yo" es el punto de transferencia de esa representación, pero simplemente no es una afirmación suficientemente fuerte decir que el "yo" está situado: el "yo", este "yo", es constituido por estas posiciones, y estas "posiciones" no son meramente productos teóricos, sino principios organizadores de prácticas materiales y arreglos institucionales profundamente incrustados, esas matrices de poder y discurso que me producen como un "sujeto" viable. Es más, este "yo" no sería un "yo" pensante y hablante si no fuera por las mismas posiciones a las que yo me opongo, pues esas posiciones, las que afirman que el sujeto se debe dar por anticipado y que el discurso es un instrumento o reflexión de ese sujeto, son ya parte de lo que me constituye. Ningún sujeto es su propio punto de partida, y la fantasía de que lo es sólo puede ser una negativa a reconocer sus relaciones constitutivas al reformularlas como el dominio de una externalidad compensatoria (1992, p. 9).

En este sentido, la identidad ya no puede ser entendida como una esencia, aquella garantía de continuidad entre un pasado, un presente y un futuro en la narración de un sujeto sobre su propia vida. Por el contrario, la identidad se reconoce en la relación entre el cambio y la permanencia, en la resistencia a cambiar en términos de lo social y lo personal, dando como resultado que los sujetos sean agentes para desarrollar, alterar e inventar su propia identidad dentro de un marco más o menos limitado de posibilidades. El sujeto así entendido es una fantasía de autogénesis. Así, en el análisis de Butler sobre la categoría de género, la autora señala que ésta solo adquiere significado a través de una serie de actos que son renovados, revisados y consolidados en el tiempo, de modo que no procuran una identidad estable, tan solo es "una identidad instituida por una repetición estilizada de actos" (Butler, 1998, p. 297). 
En consecuencia, el género ni expresa ni disfraza un 'yo' interior, pues en la medida en que se revela como una acción performativa, el acto es al mismo tiempo una ficción socialmente construida de la interioridad, en términos de concebir este 'yo' siempre situado (Butler, 1998, p. 311). El género se construye de una forma velada, pues está condicionado por las posibilidades históricas para su autogénesis, de modo que los diversos estilos de actuación se inscriben en ficciones culturales reguladas mediante castigos y sanciones en diferentes niveles. En este sentido, la propuesta de performatividad se aparta de los modelos fenomenológicos que consideran la existencia de un yo anterior a la actuación misma, de acuerdo con la lectura de Butler sobre la fenomenología ${ }^{4}$ (Triana, 2018, pp. 201-224).

Así, la propuesta de Butler se orienta a cuestionar el modelo sustancial de comprender la identidad, hacia una interpretación de la temporalidad social constituida. La identidad es instituida por los actos internamente discontinuos, una apariencia de sustancialidad como identidad constituida es tan solo el resultado performativo que remite a la repetición estilizada de actos en el tiempo; "no hay una identidad aparente de una sola pieza" (Butler, 1998, p. 301).

Lo único que queda es la repetición de la performatividad como estrategia para garantizar la apariencia del continuo identitario (Guzmán, 2017, pp. 3941). En este sentido, el problema de la narratividad como un acto, implica conforme a la propuesta de Butler la reiteración performativa de la narración, de suerte que no habría relatos falsos o verdaderos, ajustados a la realidad o distorsionados, sino pura y simplemente una actuación dramatizada -materialización- de la memoria. En consecuencia, la memoria termina por ser una capa de la identidad en la que se concreta un juego de varias piezas discontinuas. Ésta no se comprende como un conjunto de recuerdos o huellas del pasado que sean transparentes con el propio sujeto, sino que se articulan conforme a una circunstancia presente de formas muy diversas, con fallas, reescrituras, pliegues y desajustes sobre la experiencia vivida en el pasado.

4 De acuerdo con Butler (1998) la fenomenología comprende la idea de un yo previo a los actos, lo que la autora denomina Locus operativo. Sin embargo, vale la pena cuestionar si efectivamente la propuesta fenomenológica del sujeto encarnado está en la línea de la perspectiva que Butler la enuncia considerando que la crítica a la fenomenología es el supuesto para desechar la noción de expresión en privilegio de la categoría de performatividad. 


\section{La repetición desigual: perseguir las huellas de lo mismo}

La paradoja de la repetición señalada por Deleuze (2002) insiste en la identidad diferencial como imposibilidad de constituir un vínculo de reiteración de lo no idéntico (Hughes, 2009; Protevi, 2010; Rae, 2014). La desigualdad en la reiteración de actos conduce a la pregunta por cómo lograr atar en un mismo nivel de identidad actos cada vez distintos que se suceden y que se presentan como idénticos a sí mismos, tal como sugiere la regla de discontinuidad de la repetición: "el uno no aparece sin que el otro haya desparecido" (Deleuze, 2002, p. 119). La repetición se deshace al tiempo que se hace, como una carencia de sí.

Entonces, cuando se afirman como cualidades de la performance la singularidad, la desaparición y la inmediatez, éstas se pueden trasladar a la escena de la performatividad. El acto de identidad del sujeto está determinado por la reflexión en torno a las dinámicas de producción de un relato, que le da forma y lo dota de sentido. El sujeto se ve enfrentado a poner sobre un discurso los acontecimientos, experiencias y memorias sobre lo vivido para construir un aparente continuo identitario entre diversas temporalidades. No obstante, cada actuación que el sujeto elabora de sí mismo obedece a una reiteración siempre diferencial, de modo que cada narración implica la desaparición de la misma como reelaboración: "la repetición como conducta necesaria y fundamentada más que con respecto a lo que puede ser remplazado. La repetición como conducta y como punto de vista concierne a una singularidad no intercambiable, insustituible" (Deleuze, 2002, p. 27).

El sujeto no sería más que un autocreador de una actuación irrepetible y fugaz, que siempre desaparecerá en el acto mismo. Consecuencia de ello, la posibilidad de ligar las narraciones sobre la identidad se ubicará en la imposibilidad misma de volverse a actuar-narrar de manera idéntica: "la repetición es, en verdad, lo que se disfraza a la medida que se constituye, lo que no se constituye más que disfrazándose" (Deleuze, 2002, p. 44). Entonces, la pregunta por ¿quién soy yo?, remite necesariamente a una construcción narrativa que vincula la experiencia presente del sujeto con unas huellas en el pasado que le constituyen, en la medida en que le auto-definen (Derrida, 2008).

Una huella entre otras en la enunciación de la primera persona 'Yo', esto es, 'Yo soy' esta huella como el compromiso por una apuesta del presente, siempre se plantea de manera autobiográfica. El 'Yo' se llena de contenidos que se le asocian, que le constituyen y que en el mismo momento de la 
enunciación se van borrando como un pronunciamiento pasado. 'Yo soy' al mismo tiempo el heredero de una narración anterior y el hacedor de un nuevo posicionamiento que remite a las huellas siempre reelaboradas de un pasado que es inaccesible. Esto que se declara en el presente, en el instante mismo de su enunciación, revela la narración de sí mismo como una apuesta performativa o un reperformance: "lo auto-bio-gráfico se debe al hecho de que la simple instancia del 'yo' o del autos no se plantea como tal más que cuanto signo de vida, de vida en presencia, manifestación de la vida en presencia, aunque aquello que, aquel o aquella que da entonces ese signo de vida haya pasado del lado de la muerte" (Derrida, 2008, p. 73). Aunque la referencia a este 'Yo' siempre sea posible por medio de la reiteración de lo narrado, lo repetido, siempre será la muerte de ese 'Yo'.

En este sentido, la performatividad tendría como resultado el ocultamiento siempre enmascarado de lo mismo y lo otro, conforme a la paradoja de la repetición. Una máscara se sobrepone a la otra, sin que con ello exista un 'Yo' anterior a la elaboración misma, la identidad se forma en los tránsitos de enmascaramientos. Las máscaras se recubren unas a otras sin que se trate de la repetición de una primera actuación-narración, cuya repetición y reiteración mantendría la identidad. Entonces, un acto hacia la construcción de sí mismo, no será más que el ocultamiento de una identidad en otra y ninguna de ellas se reitera más que como dramatización en el teatro de operaciones. Por consiguiente, es imposible separar lo que se repite en la reiteración de la cual se forma y al tiempo se oculta, esto es, "no hay repetición pura que pueda ser abstraída o inferida del disfraz de sí. La misma cosa es disfrazante y disfrazada" (Deleuze, 2002, p. 45).

Reconocer la crisis del modelo sustancial de comprender la identidad implica también pensar que la performatividad conduce a la negación de la memoria como acontecimiento: cada acto discontinuo asegura la ficción de continuidad entre dos actos diferentes. La repetición es dentro de la performance una necesidad manifiesta para poder aprehender el registro del acto, al tiempo que desde la performatividad la repetición o reiteración del acto es la única manera de garantizar la identidad del sujeto. Sin embargo, ¿cómo es posible que la repetición de un acto anule la memoria?

Si asumimos que la naturaleza de la performatividad presentada por Butler coincide con la idea de la performance como un acto de autocreación, es posible equiparar en cierto sentido la forma en que en ambos casos opera 
la memoria. El valor indiscutible del presente, de la dramatización de una identidad o de la exhibición de cuerpo-arte que constituye un nódulo en la comprensión de la temporalidad. En ambos casos el presente es la condición de posibilidad del acto, de modo que no se produce alguno de éstos sin que se imponga la desaparición del inmediatamente anterior; el reperformance y la performatividad implican el aniquilamiento o borramiento del acto inicial. La memoria es tan solo la narración de un continuo inexistente, es la ilación de actos distintos e irrepetibles.

La memoria, en este orden de ideas, se reconoce en la misma orientación de la performatividad como una reactuación y reelaboración, en la que nunca habrá una memoria más o menos fiel con la actuación. El pasado no es una entidad fija a la cual se accede, el pasado es reflexivo y situado, la particularidad reflejada y reproducida en la que el sujeto actúa su memoria, en la que éste se esfuerza por revivir una actuación para siempre desaparecida: la memoria es un reperformance.

\section{La memoria restaurada}

Las relaciones entre performance y reperformance, entre acto original y acto repetido, entre ejecución y soporte, muestran una discusión que establece distintos niveles en la manera de construir la memoria de esta práctica artística. La idea de una ontología de la performance donde todo acto necesariamente desaparece en la ejecución revela la imposibilidad de acceder nuevamente a la experiencia de la performance, sin que al mismo tiempo se sacrifique la naturaleza del mismo. Sin duda, no es sólo una cuestión que atañe a la práctica artística de la performance, sino que puede extenderse a cualquier actividad humana: ninguna acción puede repetirse de forma idéntica.

Por ese motivo es preciso considerar que la práctica de la performance requiera eludir estas dicotomías como una manera para situarse en un marco interpretativo que permita comprender que en la reactivación de la performance existe un dinamismo entre lo nuevo y lo heredado. En efecto, reperformar es tratar de situar el performance en una atmósfera temporal que es distinta al universo de origen, si bien no es posible recuperar la performance en su versión original, restaurar la ejecución es una vía privilegiada para garantizar la documentación y la revivificación del mismo.

Así, cada actuación se presenta como un original que, no obstante, está enlazado con la ejecución anterior, es heredera de ella. La restauración de la 
performance siempre estará inscrita en un marco de significaciones de carácter cultural atada a un contexto de origen. De lo que se trata es de restaurar una actuación sin alterar la naturaleza de su práctica, esto es, reactivar la ejecución. A diferencia de los otros soportes documentales -fotografías, videos, entrevistas, etc.- la reactivación mantiene la vitalidad expresiva de la práctica. Por tanto, restaurar la acción de la performance es un esfuerzo por efectuar una nueva interpretación de la acción, semejante a la que ocurre en el teatro. Allí existe un texto que es interpretado frente a los espectadores donde los recursos de la expresividad del cuerpo en los gestos, la escenografía y la música configuran un conjunto más o menos estable de una obra que puede ser actuada y reactuada en múltiples contextos, pero que mantiene una relativa fidelidad al texto de origen.

Un clásico del teatro de William Shakespeare como Hamlet (1609) ha sido interpretado un número incontable de veces y en contextos muy disimiles, pero conserva en todas las interpretaciones una citación o repetición que asegura la identidad de la obra. El teatro tiene la capacidad de movilizar a la audiencia a actuar, puede representar y exhibir la acción, pero siempre tiene como soporte 'los fantasmas' que intervienen: "Para el padre de Hamlet convocar 'recordarme' es solicitar al joven Hamlet que actúe en su nombre, de manera que el recuerdo no está en la mente de Hamlet sino en su acto, pues todo Hamlet es un acto de memoria hecho visible" (Rayner, 2006, p. XX).

Algo semejante valdría para la práctica de la performance, en la medida en que se asume que reperformar es un 'como si' fuera posible revivir el acto original. Claramente en este contexto no se puede exigir que los/as artistas se ciñan a un guion o texto preestablecido, sino que deberán, conforme a sus recursos, mantener la vitalidad de la obra, puesto que restaurar el acto siempre está sujeto a revisión y manipulación.

No obstante, reperformar debe establecer los límites entre restaurar y remodelar. La conducta restaurada es la repetición de una materialidad -reiteración de comportamiento- que debe privilegiar la preservación del mismo. Schechner $(2011,2013)$ asegura que la conducta es un elemento externo al sujeto y que puede retrabajar, intervenir y alterar más allá de su origen en tiempo y espacio. En este sentido, el reperformance es una restauración, un 
acto que nunca se da por primera vez, se trata de una conducta que siempre es comportamiento reiterado: 'Twice-behaved behaviour's.

En conclusión, cuando se aborda la reperformance, no se debe pretender, ingenuamente, que se trata de restituir mediante la repetición un acto que es idéntico de su primera versión, como supondría la perspectiva de la ontología de la performance de Phelan, sino que es una citación o iteración de un acontecimiento pasado, entendida como una memoria restaurada. Entonces, de la misma manera que ocurre con la identidad del sujeto, la memoria queda situada en una atmósfera que siempre será constituida desde un presente determinado, cuya significación siempre está abierta a nuevas interpretaciones, tal como lo afirma Butler. Incluso, se puede ir más lejos para asegurar que la reperformación es una memoria restaurada en la que es imposible separar lo que se repite de la performance de su referente de origen, a partir del cual se forma, y a la vez se oculta, en la operación ser disfrazante y disfrazado.

\section{Referencias}

Abramovic, V.; Avgikos, J.; Iles, C.; McEvilley, T.; Obrist, H. U.; Pejic, B. \& Wulffen, T. (1998). Marina Abramovic: Artist Body. Perfomances 1969-1998. Milano: Edizioni Charta.

Banks, G. (2014). The Tensile: Repetition, Reperformance, Rearticulation. Popular Culture Association of Australia and New Zealand (PopCAANZ). Peer Reviewed Proceedings of 5th Annual Popular Culture Association of Australia and New Zealand (pp. 184-195). Hobart: PopCAANZ.

Barthold, L. S. (2014). True Identities: From Performativity to Festival. Hypatia 29(4), pp. 808-823.

Beisenbach, K. (2010). Marina Abramovic. The Artist is Present. New York: Museum of Modern Art.

Brant, H. J. (2013). "El enemigo es múltiple y adopta disfraces": La performatividad y la identidad queer en El placer desbocado de Ernesto Schoo. Hispania, 96(4), pp. 657-671.

\footnotetext{
5 “Comportamiento ejecutado dos veces". Traducción de Marcela Fuentes y Diana Taylor (2011)
} 
Butler, J. (1998). Actos performativos y constitución del género: un ensayo sobre fenomenología y teoría feminista. Debate Feminista, 18, pp. 296-314.

Butler, J., \& Scott, J. W. (1992). Feminists theorize the political. New York: Routledge.

Deleuze, G. (2002). Diferencia y repetición. Buenos Aires: Amorrortu.

Derrida, J. (2008). El animal que luego estoy si(gui)endo. Madrid: Trotta.

Fuentes, M., \& Taylor, D. (2011). Estudios avanzados del performance. México: Fondo de Cultura Económica.

Guzmán Useche, N. (2017). Frustración, deshumanización y vulnerabilidad: zoom sobre la ontología relacional de Judith Butler. Cuadernos de ética y filosofía política, 6(6), pp. 30-42.

Hughes, J. (2009). Deleuze's Difference and repetition: a reader's guide. London-New York: Continuum.

Jones, A. (2011). Posmodernismo, subjetividad y arte corporal: una trayectoria. D. Taylor \& M. Fuentes (Eds.). Estudios Avanzados de performance (pp. 125-185). México: Fondo de Cultura Económica.

Neuman, B. (1974). "Body Pressure”. Düsseldorf: Galerie Lonrad Fischer. En : Abramovic, M. (2007). Seven easy pieces. Milano: Edizioni Charta

Phelan, P. (2011). Ontología del performance: representación sin reproducción. D. Taylor \& M. Fuentes (Eds.). Estudios avanzados de performance (pp. 92-121). México: Fondo de Cultura Económica.

Protevi, J. (2010). An Approach to Difference and Repetition. Journal of Philosophy, 5(11), pp. 35-43.

Rae, G. (2014). Traces of Identity In Deleuze's Differential Ontology. International Journal of Philosophical Studies, 22(1), pp. 86-105.

Rayner, A. (2006). Ghosts: Death's Double and the Phenomena of Theatre. Minneapolis: University of Minnesota Press.

Reason, M. (2003). Archive or Memory? The Detritus of Live Performance. New Theatre Quarterly, 19(1), pp. 82-89.

Roa, C. J. C. (2015). Deleuze, el pliegue, el ritornelo y la relación arte-territorio. Cuestiones de Filosofía, 17, pp. 258-254. 
Schechner, R. (2011). Restauración de la conducta. D. Taylor \& M. Fuentes (Eds.), Estudios avanzados de performance (pp. 33-49). México: Fondo de Cultura Económica.

Schechner, R. (2013). Performance Studies: An Introduction. New York: Routledge.

Scheneider, R. (2011). In the meantime: performance remains. Performing Remains. Art and war in times of theatrical reenactment. London: Routledge and Taylor \& Francis Group.

Taylor, J. A. (2015). Reflecting subjects. passion, sympathy, and society in Hume's philosophy. Oxford: Oxford University Press.

Tomic, M. (2008). Rituals and repetitions: the displacement of context in Marina Abramovic's Seven Easy Pieces. Master of Arts Thesis. The University of British Columbia.

Triana, D. (2018). Expressivity and Performativity: The Body in its Sexual Being. Eidos, 29(29), pp. 201-224. http://rcientificas.uninorte.edu.co/index.php/eidos/article/view/9521

Vásquez, M. (2016). Fundamentos para una verdad artística: una lectura en clave filosófica de los orígenes de la producción audiovisual. Cuestiones de Filosofia, 2(19), pp. 15-30. 